the multidisciplinary team on epidural provision and developed posters for the unit on the need to respond to epidural requests within 30 minutes.

\section{EARLY ONSET OF POST-DURAL PUNCTURE HEADACHE (PDPH) AND MENINGEAL SIGNS FOLLOWING URGENT CAESARIAN SECTION. A CASE REPORT}

E Nikouli, G Karras*, B Kiamiloglou. 'Sismanogleion' General Hospital of Komotini, Komotini, Greece

\subsection{6/rapm-2021-ESRA.110}

Background and Aims 19-year-old parturient, ASA II, presented for the first time in the $\mathrm{Ob} / \mathrm{Gyn} \mathrm{ER}$, reporting intense uterine contractions, multiparous (2 previous caesarian sections), 3 days of nausea and vomit, without fever, heavy smoker, and recent food uptake.

Methods Following the first ER screening the parturient underwent spinal anaesthesia (sitting position, 25G Quincke non-traumatic needle, first try, $12 \mathrm{mg}$ chirocaine and $0.0 \mathrm{mg}$ fentanyl). Intraprocedural vomiting, lowered BP, and dizziness were observed. The caesarian section was performed uneventfully.

Results $14 \mathrm{~h}$ after the woman's re-admission, worsening headache even in prone position, nausea, tinnitus and photophobia were reported. Meningeal signs followed $24 \mathrm{~h}$ p/o. Lab tests were repeated. Blood culture, cerebral CT scan, pupilloscopy and antibiotic prophylaxis were prescribed because meningitis was suspected. There was no suspicion of an early onset of PDPH despite CT-confirmed signs of intracranial hypotension. The woman was transferred to the nearest university hospital. Neurologists diagnosed iatrogenic PDPH due to intense intracranial hypotension confirmed by cerebral CT and MRI and by excluding bacterial infection (negative blood cultures, CSF testing), and treated the patient accordingly. The continuous supine position of the woman - incompatible with a rapidly worsening PDPH - and our belief in adequate anaesthetic technique, lead us to perform PCR on the remaining blood samples for viral infection markers and they came back positive with EBV infection.

Conclusions Neurologists' early conclusion of iatrogenic $\mathrm{PDPH}, \mathrm{CT}$ images and early onset of symptoms, were in contrast with the adequate anaesthetic technique. That covered the true cause of the patient's worsening situation: meningeal irritation due to EBV infection.

\section{SPECIFIC FEATURES OF SPINAL ANESTHESIA DURING CESAREAN SECTION WITH SEVERE CORONAVIRUS PNEUMONIA}

E Oreshnikov*, S Oreshnikova, E Vasiljeva, L Kashaeva, T Denisova. Chuvash State University, Cheboksary, Russian Federation

\subsection{6/rapm-2021-ESRA.111}

Background and Aims Spinal anesthesia (SA) is the main type of anesthesia for cesarean section (SC). Severe coronavirus pneumonia, which complicates the course of pregnancy, requires some adjuncts to provide SA.

Methods The safe conduct of SA was ensured by the temporary discontinuation of the use of heparin or low molecular weight heparins in the perioperative period. SA was performed exclusively in the sitting position, then the patient was transferred to the horizontal position with the head end elevated by 30-45-60 degrees (depending on the needs). Respiratory support was used at all stages of preparation, performance, and administration of anesthesia: insufflation of humidified oxygen, high-flow oxygenation (HFO) through nasal cannula or face mask, and noninvasive mechanical lung ventilation through the face mask. Maintenance of normotension was provided by intravenous boluses of $50 \mu \mathrm{g}$ phenylephrine.

Results The above-described features of SA were used by us during CS in 30 women in labor with severe coronavirus pneumonia. Compliance with the characteristics of SA caused by coronavirus pneumonia was expressed in the following: 1) sitting position - half sitting at all stages of the perioperative period; 2) constant respiratory support, mainly HFO; 3) early transfer to the pron-position in the postoperative period, accompanied by effective postoperative anesthesia for its provision; 5) a quick return to heparin therapy in therapeutic doses. This approach ensured that there was no need to use general anesthesia with tracheal intubation for CS.

Conclusions Supplemented with HFO, half-sitting SA is the method of choice for providing CS in labor with severe coronavirus pneumonia.

\section{NEUROFIBROMATOSIS TYPE 1 AND NEUROAXIAL TECHNIQUES FOR LABOUR AND DELIVERY. ARE THEY ALWAYS FEASIBLE AND SAFE? LAST FIVE YEAR EXPERIENCE IN A TERTIARY CENTRE}

VE Vega Sanchez*, E Monge, C Corbella. Gregorio Marañon University Hospital, Madrid, Spain

\subsection{6/rapm-2021-ESRA.112}

Background and Aims Neurofibromatosis type (NF) 1 is an uncommon disease. Up to $9 \%$ of asymptomatic patients may have spinal tumours with potential enlargement during pregnancy ${ }^{1}$. Its unintentional puncture can lead to bleeding and increased intracranial pressure. Brain tumours are described; scoliosis and preeclampsia are more frequent ${ }^{2}$.

Therefore, the choice of the anaesthetic technique will be guided by risk/benefit ratio, being advisable recent neuroimaging. If any doubt, analgesia with opioids is an option or general anaesthesia if required.

Methods Gregorio Marañón University Hospital (Madrid, Spain) database was searched to identify obstetric patients with history of NF I between January 2016 and June 2021.

Results Two cases and three pregnancies were found:

A 25 -yr-old at term was attended at two labours. She had no history of neuroaxial involvement throughout time; first eutocic delivery was done under uneventful epidural anaesthesia (neuroimaging available); 2 years later required episiotomy under local anaesthesia (patient's choice).

A 39-yr-old patient with peripheral neurofibromas history and no neurological follow-up required analgesia for labor, remifentanil institutional PCA protocol was offered; but labour induction failed and c-section was done under general anaesthesia.

Conclusions Patients with NF-1 are a challenge to the obstetric anaesthesiologist and may complicate the management of labour analgesia. Neuroaxial techniques are not precluded but to be safely performed a case by case assessment and recent 
neuroimaging (brain and neuroaxial) are advisable, especially if the patient develops any neurological symptoms.

\section{AXILLARY BRANCHIAL PLEXUS BLOCK IN A WOMAN AT 34 WEEKS OF GESTATION FOR NON-OBSTETRIC DAY- SURGERY - A CASE REPORT}

E Nikouli, G Karras*, V Koutsis, F Arampatzidou, B Kiamiloglou. 'Sismanogleion' General Hospital of Komotini, Komotini, Greece

\subsection{6/rapm-2021-ESRA.113}

Background and Aims We report an axillary branchial plexus block for surgical anaesthesia in a third-trimester parturient without other comorbidities. The 30-year-old woman (34 weeks) was scheduled for external fixation of her Colles' fracture (left) after a ground-level fall. The patient consented to the proposed awake regional block technique instead of general anaesthesia.

Methods We performed a multiple-injection peripheral nerve stimulation (PNS) technique with $0.5 \%$ ropivacaine $30 \mathrm{~mL}$, $1 \%$ lidocaine $10 \mathrm{~mL}$, with 1:400.000 epinephrine as intravascular marker. The patient was placed in left lateral decubitus position with the arm to be blocked placed at a right angle to the body and the elbow flexed to 90 degrees. Skin anesthesia was also applied for the placement of an arm tourniquet. Standard monitoring was placed, supplemental oxygen was administered, baseline fetal heart rate was obtained by our obstetrical colleague. No sedatives or analgesics were administered during either procedure.

Results Our block was well tolerated and produced reliable and adequate anaesthesia during the 50-minute surgical procedure. No respiratory compromise was observed. The block lasted approximately $10 \mathrm{~h}$ and no pain was reported for $6 \mathrm{~h}$. In post-anaesthesia care unit, she had normal respiration, she did not complain about pain, and our obstetric colleagues reassessed the fetal heart rate without any new concerns. The patient was discharged the next day.

Conclusions We successfully performed adequate regional anaesthesia, using a reliable and safe technique, avoiding hemidiaphragm paralysis. This non-obstetric surgery in the parturient amplified the necessity of neuraxial anaesthesia, as branchial plexus block can be ideal for upper extremity pathology.

\section{PHEOCHROMOCYTOMA AND C-SECTION - IS REGIONAL ANAESTHESIA THE SOLUTION FOR THIS DANGEROUS COMBINATION?}

LA Montenegro Ledo, TR Alves Martins*, AR Santos Almeida Silva, MP Sanches Lucas, EC Pereira. Hospital Garcia de Orta, Almada, Portugal

\subsection{6/rapm-2021-ESRA.114}

Background and Aims Pheochromocytoma is a rare cause of hypertension during pregnancy, but it is one of the most threatening medical conditions for mother and fetus. Anaesthetic management for $\mathrm{C}$-section is challenging because it is associated with serious cardiovascular complications.

We report a successful case of c-section under combined spinal-epidural (CSE) anaesthesia in a pregnant patient with a pheocromocytoma.
Methods A 32 weeks pregnant women was referred to our hospital with persistently uncontrolled hypertension. During investigation, an abdominal MRI revealed a right-sided suprarenal mass suggesting a pheochromocytoma. Plasma and urine metanephrines were increased, confirming the diagnosis. The remain study showed normal results.

At a multidisciplinary consultation was decided to do surgical resection of pheochromocytoma after elective C-section at 36th week.

Preoperative medical preparation included $\alpha$-blockade (doxazocin), beta-blockade (propranolol) and normalization of intravascular volume (high sodium diet).

Results Before induction, a central vein and artery were cannulated for hemodynamic monitoring.

A CSE anaesthesia was performed to minimize hypertensive crises. At subarachnoid space, it was administered $11 \mathrm{mg}$ of hyperbaric bupivacaine and $3 \mathrm{mcg}$ of sufentanyl. Rapid co-filling with 11 of ringer lactate, under pressure, was administer. Adequate anaesthesia level was guaranteed and patient remained hemodynamically stable, without need of vasoactive drugs. No intercurrences were recorded.

Multimodal analgesia was prescribed for post-operative period with epidural patient control analgesia.

Conclusions Meticulous anaesthetic management is crucial and the choice of anaesthetic technique plays a decisive role in outcome of patient.

Regional anaesthesia presented as an effective technique either in blunt surgical stress and to provide post-operative analgesia.

\section{QUESTIONNAIRE SURVEY OF SATISFACTION WITH LABOUR NEURAXIAL ANALGESIA - COMBINED VERSUS EPIDURAL}

S Carvalho, A Carrão, I Ferraz*, C Castanheira, M Ghira. Hospital Beatriz Ângelo, Loures, Portugal

\subsection{6/rapm-2021-ESRA. 115}

Background and Aims Both combined spinal-epidural and epidural techniques are shown to provide effective pain relief during labour and there appears to be little basis for offering one technique over the other. The aim of this study was to evaluate if there is any difference between the techniques regarding overall maternal satisfaction.

Methods We conducted a retrospective questionnaire survey to women up to 3 days after delivery, between July and September 2020. The primary outcome was the degree of satisfaction (with a numeric rating scale from 0 to 10 ). Secondary outcomes were pain relief (with a numeric rating scale from 0 to 10) and side effects. Outcomes after labour analgesia with epidural were compared with combined spinal-epidural technique. Statistical analysis was performed using SPSS ${ }^{\circledR}$ software (version 22).

Results A total of 213 women answered the questionnaire, of which $51.6 \%$ had a combined spinal-epidural and $48.4 \%$ epidural technique. The median degree of satisfaction was 10 $[9 ; 10]$ after combined spinal-epidural and $10[8 ; 10]$ after epidural (p-value $<0,05$; figure 1). Pain relief was higher after combined spinal-epidural ( $\mathrm{p}$-value $<0,05$; figure 2 ), despite an incidence of pruritus of $60 \%$ compared with $42 \%$ after epidural (p-value <0,05). About $49 \%$ of women reported no side effects after epidural versus $31 \%$ after combined spinal- 